\title{
Nonlocal transform-domain denoising of volumetric data with groupwise adaptive variance estimation
}

\author{
Matteo Maggioni and Alessandro Foi \\ Department of Signal Processing, Tampere University of Technology, Finland \\ firstname.lastname@tut.fi
}

\begin{abstract}
We propose an extension of the BM4D volumetric filter to the denoising of data corrupted by spatially nonuniform noise. BM4D implements the grouping and collaborative filtering paradigm, where similar cubes of voxels are stacked into a four-dimensional "group". Each group undergoes a sparsifying four-dimensional transform, that exploits the local correlation among voxels in each cube and the nonlocal correlation between corresponding voxels of different cubes. Thus, signal and noise are effectively separated in transform domain. In this work we take advantage of the sparsity induced by the four-dimensional transform to provide a spatially adaptive estimation of the local noise variance by applying a robust median estimator of the absolute deviation to the spectrum of each filtered group. The adaptive variance estimates are then used during coefficients shrinkage. Finally, the inverse four-dimensional transform is applied to the filtered group, and each individual cube estimate is adaptively aggregated at its original location.

Experiments on medical data corrupted by spatially varying Gaussian and Rician noise demonstrate the efficacy of the proposed approach in volumetric data denoising. In case of magnetic resonance signals, the adaptive variance estimate can be also used to compensate the estimation bias due to the non-zero-mean errors of the Rician-distributed data.
\end{abstract}

Keywords: Volumetric data denoising, nonlocal methods, adaptive transforms, non-uniform noise, variance estimation, magnetic resonance imaging

\section{INTRODUCTION}

The most powerful methods for image restoration rely on the self-similarity and nonlocality characteristics of natural images. The state-of-the-art BM3D image denoising algorithm ${ }^{1}$ couples the nonlocal filtering paradigm proposed in $^{2,3}$ with the grouping and collaborative filtering approach. The method leverages an enhanced sparse representation in transform domain enabled by the grouping of similar 2-D image patches into 3-D data arrays which are called "groups". Collaborative filtering includes three successive steps: 3-D transformation of a group, shrinkage of transform spectrum, and inverse 3-D transformation. Due to the similarity between the grouped blocks, the transform can achieve a highly sparse representation of the true signal so that the noise can be effectively attenuated by shrinkage. In this way, the collaborative filtering reveals even the finest details shared by grouped fragments and at the same time it preserves the essential unique features of each individual fragment.

The grouping and collaborative paradigm can be also effectively exploited in volumetric data restoration and, in particular, it is the foundation of recently proposed BM4D volumetric denoising algorithm. ${ }^{4}$ Instead of using blocks of pixels as basic data patches, BM4D naturally utilizes similar 3-D cubes of voxels which are stacked together to form the 4-D group. The local correlation present among voxels in each cube as well as the nonlocal correlation between the corresponding voxels of different cubes induce a sparse representation of the group in transform domain. After collaborative filtering and inverse transformation, we obtain individual estimates of the grouped cubes, which are then aggregated at their original locations using adaptive weights.

This work was supported by the Academy of Finland (project no. 213462, Finnish Programme for Centres of Excellence in Research 2006-2011, project no. 129118, Postdoctoral Researcher's Project 2009-2011, and project no. 252547, Academy Research Fellow 2011-2016), and by Tampere Graduate School in Information Science and Engineering (also known as TISE). 
The original BM4D volumetric denoising algorithm has been utilized in the denoising of magnetic resonance (MR) images corrupted by either Gaussian- or Rician-distributed noise having uniform variance. ${ }^{4}$ However, in some applications, e.g., parallel acquisition techniques such as sensitivity encoding (SENSE) ${ }^{5}$ or generalized autocalibrating partially parallel acquisitions (GRAPPA) ${ }^{6}$ the noise corrupting the observed data is characterized by a spatially varying variance. In literature, the approaches addressing this problem generally adhere to the following scheme: at first, the variance of the noise is locally estimated, then, a filtering technique, adjusted depending on the strength of the estimated noise, is adaptively applied to the data. For example, in ${ }^{7}$ the variance is estimated using the noise distribution map and the denoising is performed via anisotropic diffusion kernels. A different approach, presented in, ${ }^{8}$ relies on the high-frequency subband of the wavelet coefficients to estimate the variance, and on a coefficients shrinkage in transform domain to filter the noisy observation. A limitation of both approaches is the assumption of data corrupted by additive zero-mean Gaussian noise. The optimized 3-D nonlocal means filter proposed in ${ }^{9}$ also addresses the Rice distribution, and it proposes to estimate the variance from the minimum distance between the high-pass components of noisy patches. This approach exploits the relation between the expectation of the squared $\ell^{2}$-distance and the variance of the noise. ${ }^{3}$

In this work, we present an extension of the BM4D denoising algorithm to data corrupted by either Gaussian or Rician noise having spatially varying variance. Exploiting the sparsity of the representation of the group in transform domain, the noise variance is accurately estimated from the outcome of robust median operations applied to the spectrum coefficients. Subsequently, the estimate is used during the collaborative filtering and the aggregation to calibrate the amount of coefficients shrinkage and the adaptive weights, respectively. Experimental results on volumetric data from the BrainWeb database demonstrate the state-of-the-art denoising performance of the proposed algorithm. In particular, our filter outperforms the method proposed in,$^{9}$ which is currently, to the best of our knowledge, the best-performing denoising method for volumetric data corrupted by spatially varying noise.

The remainder of paper is organized as follows. In Section 2 we define the adopted observation models, for both Gaussian and Rician noisy observations. Section 3 is devoted to the formal description of the fundamental steps of the algorithm, together with the techniques used to estimate the variance of the noise of both distributions. The implementation of the spatially adaptive BM4D algorithm is then formalized in Section 4. The results of the experimental validation of the proposed method are reported in Section 5, and the final discussions and general conclusions are summarized in Section 6.

\section{OBSERVATION MODELS}

\subsection{Gaussian-Distributed Noise}

We consider the noisy volumetric Gaussian observation $z_{\mathcal{N}}: X \rightarrow \mathbb{R}$ as

$$
z_{\mathcal{N}}(x)=y(x)+\eta(x), \quad x \in X,
$$

where $x=\left(x_{1}, x_{2}, x_{3}\right)$ is a 3-D coordinate belonging to the domain $X \subset \mathbb{Z}^{3}, y$ is the (unknown) original noise-free signal, and $\eta(x) \sim \mathcal{N}\left(0, \sigma^{2}(x)\right)$ is independent additive white Gaussian noise having spatially varying standard deviation $\sigma: X \rightarrow \mathbb{R}^{+}$.

\subsection{Rician-Distributed Noise}

The observation model of a Rician observation $z_{\mathcal{R}}: X \rightarrow \mathbb{R}^{+}$is

$$
z_{\mathcal{R}}(x)=\sqrt{\left(c_{r} y(x)+\sigma(x) \eta_{r}(x)\right)^{2}+\left(c_{i} y(x)+\sigma(x) \eta_{i}(x)\right)^{2}}, \quad x \in X,
$$

where $x=\left(x_{1}, x_{2}, x_{3}\right)$ is again a 3 -D coordinate belonging to the domain $X \subset \mathbb{Z}^{3}, c_{r}$ and $c_{i}$ are constants such that $0 \leq c_{r}, c_{i} \leq 1=c_{r}^{2}+c_{i}^{2}$, and $\eta_{r}(\cdot), \eta_{i}(\cdot) \sim \mathcal{N}(0,1)$ are i.i.d. random vectors following the standard normal distribution. In this way, $z_{\mathcal{R}}(x) \sim \mathcal{R}(y(x), \sigma(x))$ represents the raw magnitude MR data, modeled as a Rician distribution $\mathcal{R}$ of parameters $y$ and $\sigma: X \rightarrow \mathbb{R}^{+}$, which denote the (unknown) original noise-free signal and the spatially varying standard deviation, respectively. 


\section{BASIC ALGORITHM}

The aim of the proposed algorithm is to provide an estimate $\hat{y}$ of the original volumetric signal $y$ from the observed data $z_{\mathcal{N}}$ or $z_{\mathcal{R}}$. The proposed adaptive BM4D algorithm comprises the grouping, collaborative filtering and aggregation step as in, ${ }^{4}$ with an additional step performed after the grouping, devoted to the groupwise estimation of the noise variance.

\subsection{Grouping}

Let $C_{x_{R}}^{z}$ denote a cube of $L \times L \times L$ voxels, with $L \in \mathbb{N}$, extracted from the generic observation $z$ at the 3-D coordinate $x_{R} \in X$, which identifies its top-left-front corner. The 4-D groups are formed by stacking together, along an additional fourth dimension, 3-D cubes similar to $C_{x_{R}}^{z}$. Specifically, the similarity between two cubes is measured via the squared $\ell^{2}$-norm of the intensities difference of their voxels, normalized with respect to the size of the cube:

$$
d\left(C_{x_{i}}^{z}, C_{x_{j}}^{z}\right)=\frac{\left\|C_{x_{i}}^{z}-C_{x_{j}}^{z}\right\|_{2}^{2}}{L^{3}} .
$$

The set containing the indices of the cubes extracted from $z$ that are similar to $C_{x_{R}}^{z}$ is defined as

$$
S_{x_{R}}^{z}=\left\{x_{i} \in X: d\left(C_{x_{R}}^{z}, C_{x_{i}}^{z}\right) \leq \tau_{\text {match }}\right\},
$$

thus, two cubes are considered similar if their distance (3) is smaller or equal than a predefined threshold $\tau_{\text {match }}$. The set $S_{x_{R}}^{z}$ is consequently used to build the group associated to the reference cube $C_{x_{R}}^{z}$ as the disjoint union of the matched cubes

$$
\mathbf{G}_{S_{x_{R}}^{z}}^{z}=\coprod_{x_{i} \in S_{x_{R}}^{z}} C_{x_{i}}^{z} .
$$

Observe that each set $\mathbf{G}_{x_{R}}^{z}$ necessarily contains the reference cube $C_{x_{R}}^{z}$ because $d\left(C_{x_{R}}^{z}, C_{x_{R}}^{z}\right)=0$.

\subsection{Groupwise Variance Estimation}

We assume that the noise level in the groups (5) can be treated as constant. This is a reasonable assumption since the map $\sigma$ is typically a slowly varying function, and the grouped cubes have usually nearby coordinates. Consequently, only a single standard deviation estimate $\hat{\sigma}_{x_{R}}$ is needed for each group. We remark that a precise estimation of the variance is a crucial step during the denoising, because the amount of filtering operated on the noisy observations is proportional to the strength of the corrupting noise.

The groups are sparsely represented in transform domain as the energies of the signal and the noise are well localized in the low- and high-frequencies portions of the spectrum, respectively. Thus, an accurate groupwise variance estimation can be obtained from the median of absolute deviation ${ }^{10,11}$ (MAD) of the high-frequencies coefficients in the 4 -D group spectrum. ${ }^{12}$

\subsubsection{Gaussian-distributed data}

In case of Gaussian-distributed data $\left(z \equiv z_{\mathcal{N}}\right)$, we apply an orthonormal separable 4-D transform $\mathfrak{T}_{4 D}$ to the group (5), obtaining

$$
\Phi_{S_{x_{R}}^{z \mathcal{N}}}^{z}=\mathcal{H}\left(\mathfrak{T}_{4 D}\left(\mathbf{G}_{S_{x_{R}}^{z \mathcal{N}}}^{z_{\mathcal{N}}^{z}}\right)\right)
$$

where $\mathcal{H}$ is a high-pass filter that discards the DC hyperplane of the transform applied to the fourth dimension of the group.

A robust estimate $\hat{\sigma}_{x_{R}}$ of the standard deviation is consequently calculated as

$$
\hat{\sigma}_{x_{R}}^{z_{\mathcal{N}}}=\frac{1}{0.6745} \cdot \operatorname{MAD}\left(\Phi_{S_{x_{R}}^{z \mathcal{N}}}\right)=\frac{1}{0.6745} \cdot \operatorname{median}\left(\left\{\left|\phi_{k}-\operatorname{median}\left(\Phi_{S_{x_{R}}^{z \mathcal{N}}}\right)\right|\right\}\right), \phi_{k} \in \Phi_{S_{x_{R}}^{z \mathcal{N}}},
$$

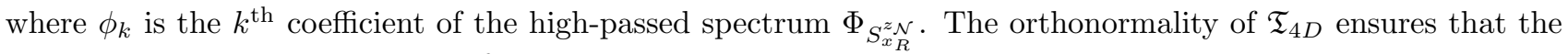
noise standard deviation in transform and spatial domain coincide. Even though this would strictly require the independence of the data, i.e. non overlapping cubes, ${ }^{12}$ we have experimentally found that the potential underestimation due to overlaps does not significantly affect the final denoising quality. 


\subsubsection{Rician-distributed data}

If the data follows the Rician distribution $\left(z \equiv z_{\mathcal{R}}\right)$, we first estimate the mean-variance pair $\left(\mu_{x_{R}}, s_{x_{R}}^{2}\right)$ of the median value of $y$ over the Rician group $\mathbf{G}_{S_{x_{R}}^{z \mathcal{R}}}^{z_{\mathcal{R}}}$ as

$$
\begin{aligned}
& \hat{\mu}_{x_{R}}=\operatorname{median}\left(\mathbf{G}_{S_{x_{R}}^{z_{\mathcal{R}}}}^{z_{\mathcal{R}}}\right), \\
& \hat{s}_{x_{R}}=\frac{1}{0.6745} \cdot \operatorname{MAD}\left(\Phi_{S_{x_{R}}^{z \mathcal{R}}}\right),
\end{aligned}
$$

where $\Phi_{S_{x_{R}}^{z \mathcal{R}}}$ is the 4-D spectrum calculated as in (6). It can be shown that from the pair $\left(\hat{\mu}_{x_{R}}, \hat{s}_{x_{R}}^{2}\right)$ one can univocally and directly obtain a robust estimate $\hat{\sigma}_{x_{R}}^{z \mathcal{R}}$ of the parameter $\sigma$ in (2).

\subsection{Collaborative Filtering}

The first phase of collaborative filtering, executed on Rician observations only, is the application of a variance stabilization transform (VST) specifically designed for the Rice distribution, ${ }^{13}$ in order to remove the dependencies between the noise and the underlying grouped data. In this way, the stabilized data can be filtered using the constant standard deviation value $c>0$ induced by the VST.

During collaborative filtering, each group is first transformed by a decorrelating separable four-dimensional transform $\mathcal{T}_{4 D}$, then the coefficients of the so-obtained spectrum are thresholded through a generic shrinkage operator $\Upsilon$ (e.g., hard thresholding or Wiener filtering) parametrized by the estimated noise level $s$. The filtered group $\hat{\mathbf{G}}_{S_{x_{R}}^{y}}^{y}$ is eventually produced by inverting the original four-dimensional transform as

$$
\mathcal{T}_{4 D}^{-1}\left(\Upsilon_{s}\left(\mathcal{T}_{4 D}\left(\mathbf{G}_{S_{x_{R}}^{z}}^{z}\right)\right)\right)=\hat{\mathbf{G}}_{S_{x_{R}}^{z}}^{y}=\coprod_{x_{i} \in S_{x_{R}}^{z}} \hat{C}_{x_{i}}^{y},
$$

where $\mathcal{T}_{4 D}$ is the combination of four 1-D linear transform that are separately applied to each dimension of the group, and $s=\hat{\sigma}_{x_{R}}^{z_{\mathcal{N}}}$ or $s=c$ if the noise is Gaussian- or Rician-distributed, respectively. The shrinkage is never applied on the DC coefficient of the 4-D spectrum, in order to preserve the mean value of the group. Each $\hat{C}_{x_{i}}^{y}$ is an estimate of the original $C_{x_{i}}^{y}$ extracted from the unknown volumetric data $y$.

Finally, in case of Rician noise, the filtered group undergoes the exact unbiased inverse variance stabilization transform as in ${ }^{13}$ that simultaneously inverts the VST and produces an unbiased estimate for the underlying $y$.

\subsection{Aggregation}

Since the cubes in the different group estimates $\hat{\mathbf{G}}_{S_{x_{R}}^{z}}^{y}$ (as well as the cubes within the same group) are likely to overlap, we may have multiple estimates for the same voxel. Therefore the final volumetric estimate $\hat{y}$ is obtained through a convex combination with adaptive weights formulated as

$$
\hat{y}=\frac{\sum_{x_{R} \in X}\left(\sum_{x_{i} \in S_{x_{R}}^{z}} w_{x_{R}} \hat{C}_{x_{i}}^{y}\right)}{\sum_{x_{R} \in X}\left(\sum_{x_{i} \in S_{x_{R}}^{z}} w_{x_{R}} \chi_{x_{i}}\right)},
$$

where each cube estimate $\hat{C}_{x_{i}}^{y}$ is assumed to be zero-padded outside its domain, and $\chi_{x_{i}}: X \rightarrow\{0,1\}$ denotes the characteristic function of the domain of a cube $\hat{C}_{x_{i}}^{y}$ located at $x_{i}$. In other words, $\chi_{x_{i}}=1$ over the coordinates of the voxels of $\hat{C}_{x_{i}}^{y}$ and $\chi_{x_{i}}=0$ elsewhere. The aggregation weights $w_{x_{R}}$ are defined to be the reciprocal of the total residual noise variance in the estimate of the corresponding groups.

\section{IMPLEMENTATION}

The general procedure described in Section 3 is implemented in two cascading stages, each composed of the grouping, noise variance estimation, collaborative filtering and aggregation steps. 


\subsection{Hard-Thresholding Stage}

In the first stage, the cubes are extracted from the generic observation $z$, and the group $\mathbf{G}_{S_{x_{R}}^{z}}^{z}$ is then formed testing the similarity measure (3) with a predefined threshold $\tau_{\text {match }}^{\text {ht }}$. After the standard deviation $\hat{\sigma}_{x_{R}}^{\text {ht }}$ of the noise is estimated from the group $\mathbf{G}_{S_{x_{R}}^{z}}^{z}$ as described in Section 3.2, collaborative filtering is realized by hard thresholding the coefficients of the spectra in (10) with an adaptive threshold value $\hat{\sigma}_{x_{R}} \cdot \lambda_{4 D}$ in case of Gaussian noise, or $c \cdot \lambda_{4 D}$ in case of Rician noise, being $c$ the value of the stabilized standard deviation. In the latter case, the group undergoes a forward and inverse VST before and after the filtering (10), respectively.

The outcome of hard-thresholding stage, $\hat{y}^{\mathrm{ht}}$, is obtained by aggregating the estimated cubes obtained obtained from collaborative filtering via the convex combination (11). The adaptive weights $w_{x_{R}}$ in (11) are reciprocal to the residual noise variance in the estimate which, in case of hard thresholding is approximated with the number $N_{x_{R}}^{\mathrm{ht}}$ of coefficients retained after thresholding times the estimated variance as

$$
w_{x_{R}}^{\mathrm{ht}}=\hat{\sigma}_{x_{R}}^{-2} N_{x_{R}}^{\mathrm{ht}^{-1}},
$$

thus penalizing groups having higher estimated variance of the corrupting noise, as well as rewarding sparser groups. Note that $N_{x_{R}}^{\mathrm{ht}} \geq 1$, since at least the DC coefficients is retained.

\subsection{Wiener-Filtering Stage}

In the Wiener-filtering stage, the grouping is performed within the hard-thresholding estimate $\hat{y}^{\text {ht }}$, thus for each reference cube $C_{x_{R}}^{\hat{y}^{\mathrm{ht}}}$ with $x_{R} \in X$ we look for similar cubes in $\hat{y}^{\text {ht }}$ via (4) using a similarity threshold $\tau_{\text {match }}^{\text {wie }}$. Since the noise is considerably reduced after the first stage, the cube-matching in $\hat{y}^{\text {ht }}$ is far more accurate. The improved correlation properties of the group are consequently beneficial to collaborative filtering because they enable a better sparsification of the data in transform domain.

The set of coordinates $S_{x_{R}}^{\hat{y}^{\mathrm{ht}}}$ is used to form two groups: one from the observation $z$, and the other from the basic estimate $\hat{y}^{\text {ht }}$, termed $\mathbf{G}_{S_{x_{R}}^{y}}^{z}$ and $\mathbf{G}_{S_{x_{R}}^{\hat{y} \mathrm{ht}}}^{\hat{y}^{\mathrm{ht}}}$, respectively. The standard deviation $\hat{\sigma}_{x_{R}}$ of the noise is estimated from the noisy data grouped in $\mathbf{G}_{S_{x_{R}}^{\text {yth }}}^{z}$, and collaborative filtering is consequently realized through an empirical Wiener filter. Element by element, the group spectrum is multiplied by the Wiener shrinkage coefficients, defined from the energy of the transformed spectrum of the basic estimate group as

$$
\mathbf{W}_{S_{x_{R}}^{\hat{y} \mathrm{ht}}}=\frac{\left|\mathcal{T}_{4 D}^{\text {wie }}\left(\mathbf{G}_{S_{x_{R}}^{\hat{y} \mathrm{ht}}}^{\hat{y}^{\mathrm{ht}}}\right)\right|^{2}}{\left|\mathcal{T}_{4 D}^{\text {wie }}\left(\mathbf{G}_{S_{x_{R}}^{\hat{y}}}^{\hat{y}^{\mathrm{ht}}}\right)\right|^{2}+s^{2}},
$$

where $s=\hat{\sigma}_{x_{R}}$ or $s=c$ when the noise follows a Gaussian or Rician distribution, respectively. As usual, the Rician-distributed data is first stabilized by a VST that shall be eventually inverted after the filtering.

The final estimate $\hat{y}^{\text {wie }}$ is produced through (11) using aggregation weights defined as

$$
w_{x_{R}}^{\text {wie }}=\hat{\sigma}_{x_{R}}^{-2}\left\|\mathbf{W}_{S_{x_{R}}^{\hat{y h t}}}\right\|_{2}^{-2},
$$

which, similarly to (12), give an estimate of the total residual noise variance of the corresponding Wiener filtered group. ${ }^{1}$

\section{EXPERIMENTS}

We evaluate the denoising performances of the proposed algorithm, termed BM4D-AV, on magnetic resonance (MR) images. As quality measure, we compute the PSNR of the denoised data as

$$
\operatorname{PSNR}(y, \hat{y})=10 \log _{10}\left(\frac{D^{2}|\tilde{X}|}{\sum_{x \in \tilde{X}}(\hat{y}(x)-y(x))^{2}}\right),
$$



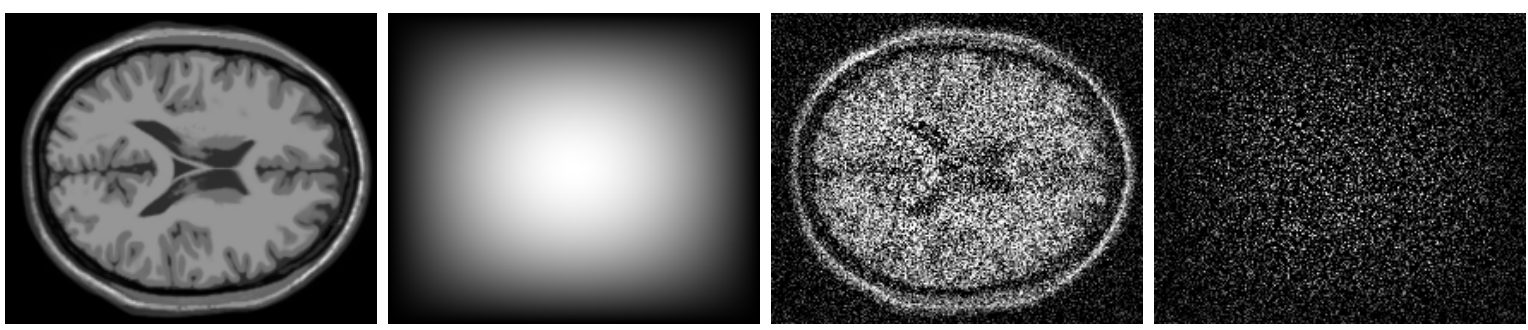

Figure 1. From left to right: original cross-section of the BrainWeb phantom; noise modulation map, with modulation factors ranging from 1 (black) to 3 (white); noisy BrainWeb phantom corrupted by Gaussian noise with standard deviation $\sigma \in[15 \% \sim 45 \%]$ varied with the modulation map; realization of the spatially varying Gaussian noise.

Table 1. Standard deviation values maximizing the PSNR denoising performance of the non-adaptive ODCT3D, ${ }^{14}$ PRINLM3D,${ }^{14}$ and $\mathrm{BM} 4 \mathrm{D}^{4}$ filters applied to the BrainWeb phantom corrupted by spatially varying noise.

\begin{tabular}{|c|c|c|c|c|c|c|c|c|c|}
\hline \multirow{2}{*}{ Noise } & \multirow{2}{*}{ ilter } & \multicolumn{8}{|c|}{$\sigma$} \\
\hline & & $1 \% \sim 3 \%$ & $3 \% \sim 9 \%$ & $5 \% \sim 15 \%$ & $7 \% \sim 21 \%$ & $9 \% \sim 27 \%$ & $11 \% \sim 33 \%$ & $13 \% \sim 39 \%$ & $15 \% \sim 45 \%$ \\
\hline \multirow{3}{*}{ Gauss. } & \multirow{3}{*}{$\begin{array}{c}\text { ODCT3D }_{\mathcal{N}} \\
\text { PRI-NLM3D } \\
{\text { BM } 4 D_{\mathcal{N}}}\end{array}$} & $2.33 \%$ & $6.86 \%$ & $11.34 \%$ & $15.62 \%$ & $19.91 \%$ & $24.70 \%$ & $29.01 \%$ & $33.61 \%$ \\
\hline & & $2.35 \%$ & $6.39 \%$ & $10.90 \%$ & $15.11 \%$ & $19.90 \%$ & $23.39 \%$ & $28.47 \%$ & $32.83 \%$ \\
\hline & & $2.25 \%$ & $6.98 \%$ & $11.62 \%$ & $16.80 \%$ & $21.60 \%$ & $27.22 \%$ & $32.17 \%$ & $38.25 \%$ \\
\hline \multirow{3}{*}{ Rician } & \multirow{3}{*}{$\begin{array}{c}\text { ODCT3D }_{\mathcal{R}} \\
\text { PRI-NLM } 3 \mathrm{D}_{\mathcal{R}} \\
{\text { BM } 4 \mathrm{D}_{\mathcal{R}}}\end{array}$} & $2.33 \%$ & $5.96 \%$ & $69 \%$ & $12.64 \%$ & $15.69 \%$ & $18.88 \%$ & $22.37 \%$ & $25.27 \%$ \\
\hline & & $2.18 \%$ & $5.96 \%$ & 9. & 12 & $9 \%$ & 19 & $22.23 \%$ & $25.49 \%$ \\
\hline & & $2.25 \%$ & $6.30 \%$ & $9.37 \%$ & $12.86 \%$ & $15.52 \%$ & $19.39 \%$ & $22.42 \%$ & $25.87 \%$ \\
\hline
\end{tabular}

where $D$ is the peak of $y, \tilde{X}=\{x \in X: y(x)>10 \cdot D / 255\}$ (in order not to compute the PSNR on the background as $\operatorname{in}^{14}$ ), and $|\tilde{X}|$ is the cardinality of $\tilde{X}$. Additionally, we evaluate the results of denoising via the 3 -D extension of the structure similarity index (SSIM) ${ }^{14,15}$ which should better agree with subjective perceptual quality.

The observations used in our experiments are corrupted by either Gaussian or Rician noise, and the volumetric test data is the T1 brain phantom of size $181 \times 217 \times 181$ voxels from the BrainWeb database. ${ }^{16}$ According to (1) and (2), we synthetically generate the noisy observations $z_{\mathcal{N}}$ and $z_{\mathcal{R}}$ by adding spatially varying Gaussian and Rician noise having different ranges of standard deviation $\sigma$, expressed as percentage of the maximum value of the signal $y$. Specifically, we first generate a realization of Gaussian or Rician noise with uniform standard deviation $\sigma$, then we multiply each sample of such realizations by a volumetric noise modulation map as in, ${ }^{9}$ which smoothly increases the amount of noise from the extrema to the center of the volume up to a factor of 3. Figure 1 illustrates an example of noisy observation obtained by a modulated Gaussian noise with varying standard deviation $\sigma \in[15 \% \sim 45 \%]$.

As a comparison, we validate the denoising performances of the proposed BM4D algorithm against the optimized adaptive blockwise nonlocal means OB-AR-NLM3D-WM. ${ }^{9}$ The BM4D is tuned as proposed in, ${ }^{4}$ and the noise-variance estimation and the collaborative filtering steps use the same transform, i.e. $\mathcal{T}_{4 D} \equiv \mathfrak{T}_{4 D}$, both in the hard-thresholding and Wiener-filtering stages. In this way, the groups need to be transformed only once. We also present the performances of the current best-performing non-adaptive methods. In particular, we test the BM4D, ${ }^{4}$ ODCT3D,${ }^{14}$ and PRI-NLM3D ${ }^{14}$ filters, using constant standard deviation values found maximizing the restored quality in terms of PSNR. Table 1 reports the optimum values of standard deviation used by the three non-adaptive algorithms during the denoising of the BrainWeb phantom corrupted by spatially varying noise having eight different ranges of standard-deviation. Moreover, we present the results obtained by an Oracle filter, namely the state-of-the-art BM4D, ${ }^{4}$ having exact knowledge on the varying standard deviation $\sigma(x)$ for each $x \in X$.

As one can clearly see, both the objective performances reported in Table 2 and the visual appearance of the denoised phantoms shown in Figure 2, substantiate the superior quality of the results produced by the proposed BM4D-AV. In particular, BM4D-AV outperforms the state-of-the-art adaptive filter OB-AR-NLM3D-WM and the non-adaptive state-of-the-art filter BM4D with PSNR improvements of up to $2.5 \mathrm{~dB}$ and $0.5 \mathrm{~dB}$ in case of Gaussian observations, and about $0.2 \mathrm{~dB}$ in case of Rician observations. Let us remark that BM4D-AV performs 
Table 2. PSNR (left value in each cell) and $\operatorname{SSIM}^{15}$ (right value in each cell) denoising performances on the volumetric test data from the BrainWeb database ${ }^{16}$ of the non-adaptive ODCT3D, ${ }^{14}$ PRI-NLM3D,${ }^{14}$ BM$^{4} D^{4}$ filters, the adaptive OB-AR-NLM3D-WM ${ }^{9}$ filter, and the proposed adaptive BM4D-AV (tuned with the modified profile as in ${ }^{4}$ ). Two kinds of observations are tested, corrupted by spatially varying Gaussian and Rician noise, synthetically generated according to the observation models (1) and (2), respectively. Both cases are tested under different ranges of standard-deviations $\sigma$, expressed as percentage relative to the maximum intensity value of the original volumetric data. The ranges of variation for $\sigma$ are shown in the header of the table. The PSNR and SSIM values of the noisy data, and of the denoised phantoms produced by an Oracle, namely BM4D with exact knowledge of the varying $\sigma$, are also shown for comparison. The subscripts $\mathcal{N}$ (Gaussian) and $\mathcal{R}$ (Rician) denote the addressed noise distribution.

\begin{tabular}{|c|c|c|c|c|c|c|c|c|c|}
\hline \multirow{2}{*}{ Noise } & \multirow{2}{*}{ lter } & \\
\hline & & $1 \% \sim 3 \%$ & $3 \% \sim 9 \%$ & $5 \% \sim 15 \%$ & $7 \% \sim 21 \%$ & $9 \% \sim 27 \%$ & $11 \% \sim 33 \%$ & $13 \% \sim 39 \%$ & $15 \% \sim 45 \%$ \\
\hline \multirow{7}{*}{ Gauss. } & & 3410 & .62 & 44 & .33 & $5.26 \mid 0.25$ & $3.51 \mid 0.20$ & $12.06 \mid 0.16$ & $10.82 \mid 0.13$ \\
\hline & \multirow{3}{*}{$\begin{array}{c}\text { ODCT3D }_{\mathcal{N}} \\
\text { PRI-NLM3 } 3 \mathrm{D}_{\mathcal{N}} \\
{\text { BM } 4 \mathrm{D}_{\mathcal{N}}}\end{array}$} & $.04 \mid \mathbf{0 . 9 8}$ & .94 & $31.43 \mid 0.90$ & .86 & $28.42 \mid 0.83$ & $27.40 \mid 0.80$ & $26.52 \mid 0.77$ & $25.74 \mid 0.74$ \\
\hline & & $0.71 \mid 0.98$ & $34.50 \mid 0.94$ & $31.75 \mid 0.91$ & 29.95 & $28.60 \mid 0.83$ & $27.49 \mid 0.80$ & $26.55 \mid 0.77$ & $25.79 \mid 0.74$ \\
\hline & & $0.42 \mid \mathbf{0 . 9 8}$ & 34.9 & 32.5 & .89 & $29.91 \mid \mathbf{0 . 8 7}$ & $28.99 \mid \mathbf{0 . 8 5}$ & $28.23 \mid \mathbf{0 . 8 3}$ & $27.56 \mid \mathbf{0 . 8 1}$ \\
\hline & \multirow{2}{*}{$\begin{array}{c}\text { OB-AR-NLM3D-WM } \\
\text { BM4D-AV } \\
\mathcal{N}\end{array}$} & $40.38 \mid \mathbf{0 . 9 8}$ & 34.5 & 31.5 & 29. & 28.1 & $26.89 \mid 0.73$ & $25.86 \mid 0.68$ & $24.95 \mid 0.64$ \\
\hline & & $40.45 \mid \mathbf{0 . 9 8}$ & 35.4 & 33.1 & 0.90 & 30.24 & $|29.22| 0.85$ & 0.82 & $|\mathbf{2 7 . 5 9}| 0.79$ \\
\hline & & $40.96 \mid 0.98$ & $35.56 \mid 0.96$ & $33.14 \mid 0.93$ & 0.91 & $30.36 \mid 0.88$ & $29.40 \mid 0.86$ & $28.58 \mid 0.84$ & $27.87 \mid 0.82$ \\
\hline \multirow{7}{*}{ Rician } & & 0.90 & 24 & 20.0 & & 15. & 19 & 15 & $\begin{array}{l}11.04 \mid 0.12 \\
\end{array}$ \\
\hline & ODC & $39.70 \mid \mathbf{0 . 9 8}$ & $\begin{array}{l}33.13 \mid 0.92 \\
\end{array}$ & \begin{tabular}{|l|}
$29.58 \mid 0.86$ \\
\end{tabular} & 26.9 & $24.72 \mid \mathbf{0 . 7 4}$ & $\mid$\begin{tabular}{|l|}
$22.85 \mid \mathbf{0 . 7 0}$ \\
\end{tabular} & \begin{tabular}{|l|l|}
$21.12 \mid \mathbf{0 . 6 6}$ \\
\end{tabular} & \begin{tabular}{|l|l|}
$19.47 \mid \mathbf{0 . 6 2}$ \\
\end{tabular} \\
\hline & PRI-NLM3 D $_{\mathcal{R}}$ & $40.53 \mid 0.98$ & $33.21 \mid 0.93$ & $29.29 \mid 0.87$ & $26.21 \mid 0.80$ & $23.71 \mid \mathbf{0 . 7 4}$ & $21.68 \mid 0.69$ & $19.88 \mid 0.65$ & $18.21 \mid 0.61$ \\
\hline & $\mathrm{BM} 4 \mathrm{D}_{\mathcal{R}}$ & $40.34 \mid \mathbf{0 . 9 8}$ & $33.76 \mid 0.93$ & $30.19 \mid 0.86$ & $|27.37| 0.80$ & $24.95 \mid 0.73$ & $22.89 \mid 0.68$ & $21.07 \mid 0.63$ & $19.39 \mid 0.59$ \\
\hline & \multirow{2}{*}{\begin{tabular}{|c} 
OB-AR-NLM3D-WM $\mathrm{WM}_{\mathcal{R}}$ \\
BM4D-AV $\mathrm{AV}_{\mathcal{R}}$ \\
\end{tabular}} & $40.28 \mid \mathbf{0 . 9 8}$ & $34.29 \mid \mathbf{0 . 9 4}$ & $31.16 \mid 0.87$ & $28.73 \mid 0.81$ & $26.43 \mid \mathbf{0 . 7 4}$ & $24.17 \mid 0.67$ & $22.00 \mid 0.60$ & $20.00 \mid 0.54$ \\
\hline & & $40.43 \mid \mathbf{0 . 9 8}$ & $34.41 \mid 0.94$ & $31.27 \mid 0.89$ & \begin{tabular}{|l|l|}
28.80 & 0.82 \\
\end{tabular} & $26.55 \mid 0.74$ & \begin{tabular}{|l|l|}
$\mathbf{2 4 . 2 1}$ & 0.67 \\
\end{tabular} & \begin{tabular}{|l|l|}
$\mathbf{2 2 . 1 1}$ & 0.61 \\
\end{tabular} & $\mathbf{2 0 . 0 1 | 0 . 5 6}$ \\
\hline & Oracle $_{\mathcal{R}}$ & $40.90 \mid 0.98$ & $34.85 \mid 0.95$ & $31.59 \mid 0.91$ & $28.99 \mid 0.85$ & $26.82 \mid 0.76$ & $24.55 \mid 0.70$ & $22.43 \mid 0.65$ & $20.37 \mid 0.61$ \\
\hline
\end{tabular}

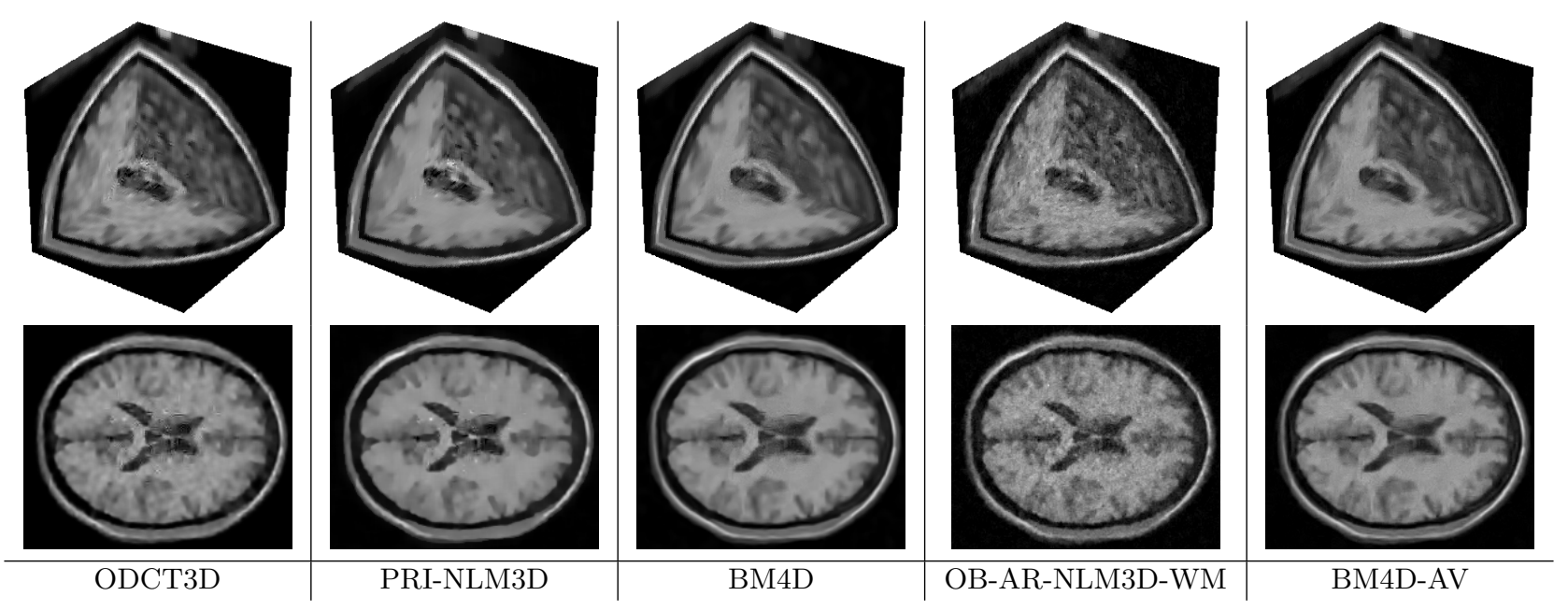

Figure 2. From left to right, denoising results of the ODCT3D, PRI-NLM3D, BM4D, OB-AR-NLM3D-WM, and the proposed BM4D-AV filter applied to the BrainWeb phantom corrupted by spatially varying Gaussian noise with standard deviation $\sigma \in[15 \% \sim 45 \%]$. The original and corrupted data can be seen in Figure 1. For each algorithm, both the 3-D and 2-D transversal cross-section of the phantom are presented in the top and bottom row, respectively. 
only marginally worse than the Oracle filter, which, not surprisingly, always achieves the best performances. Figure 2 confirms the objective results. We observe that the denoised phantom produced by OB-AR-NLM3DWM is considerably affected by residual noise, thus suggesting that the variance is underestimated during the filtering. The non-adaptive filters behave reasonably well, even though the effects of a fixed level of noise are clearly visible. In particular, the center of the phantom is under-smoothed as the applied amount of filtering is not sufficient to completely remove the noise. Conversely, the peripheral areas are over-smoothed.

\section{CONCLUSIONS}

Experiments show that the proposed adaptive BM4D-AV achieves state-of-the-art performances in volumetric data denoising under condition of spatially varying Gaussian- or Rician-distributed noise in terms of objective (Table 2) and subjective visual (Figure 2) quality. The groupwise noise estimation embedded in the proposed BM4D-AV allows for a correct filtering of the noisy data in any section of the phantom. As a matter of fact, our filter is able to simultaneously preserve the edges of fine details and the smoothness of flat areas. We also wish to remark that the proposed algorithm exhibits the most gentle performance decay as the level of noise increases. Thus, BM4D-AV can be a viable and effective tool in medical image processing when there is no precise knowledge about the statistics of the noise corrupting the observed data.

\section{REFERENCES}

[1] Dabov, K., Foi, A., Katkovnik, V., and Egiazarian, K., "Image denoising by sparse 3D transform-domain collaborative filtering," IEEE Transactions on Image Processing 16, 2080-2095 (August 2007).

[2] De Bonet, J. S., "Noise reduction through detection of signal redundancy," tech. rep., Rethinking Artificial Intelligence, MIT AI Lab (1997).

[3] Buades, A., Coll, B., and Morel, J., "A non-local algorithm for image denoising," in [Proceedings of the 2005 IEEE Computer Society Conference on Computer Vision and Pattern Recognition], 2, 60-65 (2005).

[4] Maggioni, M., Katkovnik, V., Egiazarian, K., and Foi, A., "A nonlocal transform-domain filter for volumetric data denoising and reconstruction," submitted to IEEE Transactions on Image Processing (2011).

[5] Pruessmann, K. P., Weiger, M., Scheidegger, M., and Boesiger, P., "SENSE: Sensitivity encoding for fast MRI," Magnetic Resonance in Medicine 42, 952-962 (1999).

[6] Griswold, M., Jakob, P., Heidemann, R., Nittka, M., Jellus, V., Wang, J., Kiefer, B., and Haase, A., "Generalized autocalibrating partially parallel acquisitions (GRAPPA)," Magnetic Resonance in Medicine 47(6), 1202-1210 (2002).

[7] Samsonov, A. and Johnson, C., "Noise-adaptive nonlinear diffusion filtering of MR images with spatially varying noise levels," Magnetic Resonance in Medicine 52, 798-806 (October 2004).

[8] Delakis, I., Hammad, O., and Kitney, R. I., "Wavelet-based de-noising algorithm for images acquired with parallel magnetic resonance imaging (MRI)," Physics in Medicine and Biology 52(13), 3741 (2007).

[9] Manjón, J. V., Coupé, P., Martí-Bonmatí, L., Collins, D. L., and Robles, M., "Adaptive non-local means denoising of MR images with spatially varying noise levels," Journal of Magnetic Resonance Imaging 31, 192-203 (2010).

[10] Hampel, F., "The influence curve and its role in robust estimation," Journal of the American Statistical Association 69, 383-393 (June 1974).

[11] Donoho, D., Johnstone, I., and Johnstone, I., "Ideal spatial adaptation by wavelet shrinkage," Biometrika 81(3), 425-455 (1993).

[12] Danielyan, A. and Foi, A., "Noise variance estimation in nonlocal transform domain," in [International Workshop on Local and Non-Local Approximation in Image Processing (LNLA)], 41-45 (August 2009).

[13] Foi, A., "Noise estimation and removal in MR imaging: the variance-stabilization approach," in [Proceedings of the IEEE International Symposium on Biomedical Imaging: From Nano to Macro], (2011).

[14] Manjón, J. V., Coupé, P., Buades, A., Collins, D. L., and Robles, M., "New methods for MRI denoising based on sparseness and self-similarity," Medical Image Analysis (in press) (2011).

[15] Wang, Z., Bovik, A., Sheikh, H., and Simoncelli, E., "Image quality assessment: from error visibility to structural similarity," IEEE Transactions on Image Processing 13, 600-612 (April 2004).

[16] Vincent, R., "Brainweb: Simulated brain database." http://mouldy.bic.mni.mcgill.ca/brainweb/ (2006). 